\title{
¿Garantes de la salvaguarda del aragonés? Situación y resultados de la enseñanza del aragonés implementada desde 1997
}

\author{
I. O. CAMPOS BANDRÉS \\ Universidad de Zaragoza
}

Recibido: 10 octubre 2014 / Aceptado: 9 noviembre 2014

ISBN: $1697-7467$

\begin{abstract}
RESUMEN: Uno de los principales retos de la sociedad actual es la adaptación a los nuevos contextos comunicativos. Este hecho ha derivado en la primacía de algunos idiomas internacionales en el ámbito educativo. Mientras algunos territorios han conseguido que la entrada de estos idiomas no conlleve la desatención a sus lenguas propias, la administración aragonesa todavía apuesta por un modelo que prima la lengua castellana y las extranjeras. Interesados por aportar los primeros datos científicos sobre los resultados de la enseñanza del aragonés, evaluamos la competencia lingüística de su alumnado, encontrando el escaso dominio que permite alcanzar el obsoleto modelo actual.
\end{abstract}

Palabras clave: competencia lingüística, aragonés, lenguas propias, L2.

Aragonese language 'keepers'? The teaching of Aragonese implemented since 1997: current situation and prospects

\begin{abstract}
One of the main challenges of XXIst society is the adaptation to new communicative contexts. This fact has resulted in a great impulse of the teaching of and in some international majority languages. Some plurilingual territories have accomplished a balanced situation by the use of the regional and the majority languages as a medium of instruction. Nevertheless, Aragonese government is developing mainly the foreign and the state language teaching. With the purpose of make progress in the results of its teaching, we have evaluated the linguistic competence of Aragonese students. Results have shown the very little competence achieved by students thanks to this obsolete system.
\end{abstract}

Keywords: linguistic compentece, Aragonese, regional languages, L2.

\section{INTRODUCCIÓN}

El aragonés es una de las dos lenguas propias de la Comunidad Autónoma de Aragón que en la actualidad sobrevive en algunos territorios del norte de la provincia de Huesca. A falta de un censo oficial de hablantes, las cifras del censo de habitantes de 1981 (López, 2013), en el cual se incluyó una pregunta respecto a las lenguas propias de la Comunidad, reflejaba un total de 11.284 hablantes activos de aragonés y de 17.653 hablantes pasivos, es decir, personas capaces de comprenderlo pero no de comunicarse en esta lengua. Los datos del posterior estudio de Llera (2001) recogían un 45,4\% de altoaragoneses hablantes pasivos y un $28,5 \%$ de altoaragoneses que hablaban el aragonés, aunque solo en $4,6 \%$ manifestaba 
ser capaz de escribirlo. Estos datos muestran una realidad incuestionable: el aragonés se encuentra en una situación de empleo minoritario y la mayor parte de los aragonesohablantes son analfabetos en su lengua materna. Todo ello es consecuencia de siglos de supervivencia del aragonés en situación diglósica.

Durante las primeras décadas del siglo XX, la escolarización obligatoria en castellano culminó un cúmulo de factores desfavorables para el mantenimiento del aragonés, creando "el problema del lenguaje contrariado" (Satué y L’Hotellerie, 2011) que suponía para los escolares la ruptura entre el lenguaje familiar de su vida cotidiana y aquel en el que se les exigía aprender en la escuela. Esta prohibición que se ha impuesto a lo largo del tiempo a los hablantes nativos de aragonés de aprender y ser alfabetizados en su propia lengua en la escuela ha favorecido lo que Skutnabb-Kangas (2009) denomina como genocidio lingüistico.

A pesar de que desde de la segunda mitad del siglo XX, pero sobre todo durante las últimas décadas, se ha asumido completamente que el bilingüismo o, más bien, el mutilingüismo ha sido y es a nivel mundial una realidad mucho más frecuente que el monolingüismo (Grosjean, 1982; Cook, 1992; Mackey, 1967), lo cierto es que muchos estados y territorios, como el aragonés, todavía no han dado respuesta a su realidad plurilingüe a través sus sistemas educativos. De este modo postergan unos modelos de enseñanza monolingües nacidos a mediados del siglo XIX como consecuencia del auge de los estados-nación y cuyo interés era la instrucción en la lengua única como garantía de la unidad nacional (Siguán, 2001).

La exterminación de las lenguas propias fue un objetivo común a todos los países de la actual Europa, dentro de un periodo de minorización de las lenguas no oficiales (Vila, 1994) que se extendió entre 1850 y 1950 y en el que la persecución y denigración de los hablantes de lenguas propias en el sistema educativo fue una práctica extendida. Con todo y con eso, tras la II Guerra Mundial la situación fue cambiando paulatinamente, gracias al desgaste de los ideales nacionalistas, la evolución de la investigación en el área de la psicología aplicada a la adquisición de lenguas y los movimientos de reivindicación impulsados por culturas dominadas como la bretona, catalana, flamenca, gala o frisona. Así, se ha ido produciendo un mayor reconocimiento y dignificación de las ahora conocidas como lenguas regionales de Europa tanto por parte de los diferentes estados como de las propias instituciones europeas (Comisión de las Comunidades Europeas, 2008; Consejo de Europa, 1992, 2008, 2010).

Sin embargo, en algunos territorios esta toma de conciencia todavía no se ha dado y aunque la enseñanza de lenguas extranjeras sí ha sido asumida y potenciada, las lenguas propias siguen en un estado de exterminio y asimilación por parte de la lengua mayoritaria/ estatal. Este es el caso de la Comunidad Autónoma de Aragón, territorio con una realidad histórica trilingüe que ha desatendido su riqueza lingüística a lo largo de la historia.

Son muchas las experiencias dentro y fuera del Estado que hubieran podido servir de referencia a la Comunidad aragonesa para el desarrollo de sistemas de enseñanza que garantizasen la salvaguarda de las lenguas propias, con los modelos bilingües de mantenimiento e inmersión lingüística como ejemplo más efectivo para la protección de la lengua y la generación de nuevos hablantes. El ejemplo paradigmático de este tipo de enseñanza es el canadiense (Lambert y Tucker, 1972), pero también han sido importantes referentes comunidades vecinas como la catalana y la vasca (Cenoz, 2009; Vila, 2008), u otras del Estado como la gallega (Loredo et al, 2007). También las experiencias de Escocia (Johnstone, 2006), Frisia (Ytsma, 2007), el País de Gales (Lewis, 2008) o Noruega (Hirvonen, 2008) 
podrían haber sido modelos interesantes para el territorio aragonés. Asimismo, trabajos como el de Piquer (2006) demuestran la viabilidad en el desarrollo de proyectos lingüísticos que garanticen la adquisición de la lengua propia, la estatal y la extranjera siendo todas ellas vehiculares dentro del aula desde el inicio de la escolaridad.

Sin embargo, en el caso aragonés la tradición minorizadora y asimilacionista ha prevalecido sobre los resultados constatados desde el ámbito científico e incluso sobre las indicaciones europeas (Consejo de Europa, 1992; Unesco, 1996) y la propia legislación autonómica (Justicia de Aragón, 1993; Cortes de Aragón, 2007).

A lo largo de los últimos años, los expertos (López, 2013; Nagore, 2001) pero también los propios hablantes nativos de aragonés (Castán, 1998) han advertido el descenso del número de hablantes y la ruptura en la transmisión intergeneracional de la lengua. Por otra parte, han sido bastantes las voces que desde 1984, cuando el catalán llegó a las aulas aragonesas de la Franja Oriental (DGA, 2009), han solicitado la entrada del aragonés en la escuela (Quintana, 1985), entendiendo que su enseñanza debía implementarse a través de modelos de mantenimiento e inmersión lingüística (Alcover y Quintana, 2000). Sin embargo no ha sido hasta 2014, con la llegada de la LOMCE y la necesaria reestructuración de la enseñanza en la Comunidad Autónoma, cuando se ha aprobado un currículum para la enseñanza del aragonés, que en cualquier caso supone la continuidad de la enseñanza tal y como se ha desarrollado hasta hoy: como asignatura optativa durante una hora a la semana.

Encontramos muchos ejemplos en Europa que apuntan al fracaso de modelos en los que la lengua propia ha entrado en el sistema educativo pero no como lengua vehicular, como el caso de Irlanda (Ó Laoire, 2007) o de las comunidades autónomas españolas que implementaron los Decretos de Bilingüismo a principios de 1978 (Siguán, 2001).

Así, mientras en otros territorios plurilingües se implementa la enseñanza multilingüe para satisfacer las demandas de una sociedad globalizada que precisa del profundo conocimiento de lenguas extranjeras (Cenoz, 2009), en Aragón se apuesta por un modelo obsoleto en el que tan solo la lengua estatal y las extranjeras son vehículo de la enseñanza (Consejería de Educación del Gobierno de Aragón, 2013; Departamento de Educación, Universidad, Cultura y Deporte del Gobierno de Aragón, 2014).

Revisado el estado de la cuestión, la pregunta de investigación que se nos presentó fue: ¿es el tipo de enseñanza del aragonés desarrollado desde 1997 hasta la actualidad suficiente para propiciar un relevo generacional que garantice la salvaguarda de esta lengua?

Con el fin de aportar los primeros datos al respecto desarrollamos una experiencia piloto de naturaleza exploratoria, centrada en la competencia lingüística del alumnado de aragonés de $2^{\circ}$ ciclo de Educación Primaria de tres centros altoaragoneses con diferentes realidades sociolingüísticas: 1) situado en un lugar donde el aragonés es lengua materna de la mayor parte del alumnado y la asignatura se imparte fuera del horario escolar, 2) situado en zona de empleo histórico del aragonés pero en donde no se habla actualmente y con la asignatura dentro del horario escolar, y 3) situado en zona de empleo histórico del aragonés pero en donde no se habla actualmente y con la asignatura fuera del horario escolar.

Dada la naturaleza exploratoria de nuestro estudio, no partimos de hipótesis sino de una serie de presupuestos de partida que tras la obtención de resultados podrían derivar en hipótesis a contrastar en un futuro estudio llevado a cabo con una muestra representativa. Nuestros presupuestos de partida eran: 1) La enseñanza actual del aragonés no garantiza una competencia lingüística suficiente entre el alumnado de territorios de empleo histórico de la 
lengua; y 2) En los territorios de empleo histórico pero no actual de la lengua, su presencia en el horario escolar influirá de un modo positivo en el nivel de competencia del alumnado.

\section{MÉtodo}

\subsection{Participantes}

Dado que la situación sociolingüística de la lengua aragonesa es muy diversa, consideramos necesario ordenar los centros en los que se enseña bajo tres tipologías: aquellos situados en lugares donde es lengua materna (Tipo - L1), aquellos situados en lugares de empleo histórico pero donde se enseña en horario escolar (Tipo L2 - escolar), y aquellos situados en lugares de empleo histórico pero donde se enseña en horario extraescolar (Tipo L2 - extraescolar). Con ello pretendíamos saber, en primer lugar, si es el factor "enseñanza" (tal y como se desarrolla actualmente) suficiente para garantizar la competencia en la lengua en el territorio no aragonesófono y, en segundo lugar, si es el factor "horario lectivo" influyente sobre el grado de competencia adquirida, pues podría darse el caso de que la dignidad que aporta a la lengua su enseñanza en horario escolar influyera en las actitudes $\mathrm{y}$, por ende, en la competencia adquirida por el alumnado.

Durante el curso 2013/2014 había ocho escuelas bajo la tipología 1, tres de Tipo L2 escolar y diez de Tipo L2 - extraescolar.

Dado que el número de alumnos que cursan aragonés supone una muestra muy reducida, siendo durante el curso 2012/2013 un total de 387 (Consejo Escolar de Aragón, 2014) el objetivo final es realizar la evaluación sobre todo el universo de $2^{\circ}$ ciclo, siendo la investigación que aquí presentamos un estudio piloto de carácter exploratorio.

Teniendo en cuenta el carácter exploratorio de este estudio piloto, para la selección de los participantes se estimó oportuno un muestreo no probabilístico, de tipo estratégico. De este modo, teniendo en cuenta la categorización establecida para los centros donde se cursa la asignatura de aragonés (Tipo 1 - L1, Tipo L2 - escolar y Tipo L2 - extraescolar) se seleccionó el centro de cada uno de los tres tipos que tenía un mayor número de alumnos de $2^{\circ}$ ciclo cursando lengua aragonesa.

En los centros de Tipo L2 - escolar y Tipo L2 - extraescolar la prueba se realizó con el alumnado de $2^{\circ}$ ciclo que cursaba aragonés, pero dentro de la escuela del centro Tipo 1 se seleccionó a todo el alumnado de $2^{\circ}$ ciclo de Educación Primaria, dado que uno de los objetivos de nuestra investigación era comprobar si la enseñanza de la lengua propia producía diferencias en la competencia del alumnado que cursa esta asignatura y el que no la cursa en un contexto de bilingüismo social.

Cabe señalar que en un primer momento se pretendía realizar esta prueba con alumnos de $3^{\circ}$ ciclo pero la escasez de alumnado de esta etapa cursando aragonés en la mayor parte de los centros (en concreto en el total de los centros de Tipo L2 - escolar solo había un alumno) nos llevó a realizar esta leve modificación en nuestro estudio.

Finalmente contamos con 12 alumnos del centro de Tipo 1, 6 alumnos del centro de Tipo L2 - escolar, y 13 alumnos del centro de Tipo 2 - extraescolar, de los cuales dos pruebas fueron desestimadas por haber sufrido sesgos durante su aplicación, por lo que el tratamiento de los datos quedó reducido a las pruebas de 11 sujetos en este centro. 


\subsection{Instrumento}

El instrumento seleccionado fue la prueba de evaluación de diagnóstico elaborada por el Departamento de Educación del Gobierno de Aragón para el área de inglés en el curso académico 2009/2010, para segundo ciclo de Educación Primaria. Este examen fue adaptado a la lengua aragonesa y validado por jueces.

La prueba seleccionada está diseñada para la medición de la competencia lingüística del alumnado desde la perspectiva comunicativa, entendiendo que esta es la que "se pone en funcionamiento con la realización de distintas actividades de la lengua que comprenden la comprensión, expresión, interacción o mediación (interpretando o traduciendo) con textos orales y/o escritos" (Departameto de Educación, Cultura y Deporte del Gobierno de Aragón, 2009).

Cabe señalar que la adaptación de la prueba de lengua inglesa a la lengua aragonesa supone la reducción del nivel evaluado dada la proximidad de los sistemas lingüísticos castellano y aragonés. Con todo y con eso, se consideró oportuno aplicarla sin aumentar el nivel tras su traducción dada la diferencia horaria que existe entre la enseñanza del inglés (3 horas semanales) y del aragonés (1 hora semanal) en los centros participantes.

El cuestionario original contaba con tres apartados centrados en la evaluación de la competencia en comprensión oral, en comprensión escrita y en gramática, para los cuales se precisaba un análisis de tipo cuantitativo.

Se decidió incluir un cuarto apartado para la evaluación de la expresión escrita con el fin de obtener unos resultados más completos, que se analizó desde una perspectiva cualitativa. Para diseñar esta prueba se tomaron como referencia los estándares del Marco Común Europeo de Referencia para las Lenguas y en concreto los referidos al nivel A1/A2: "Soy capaz de escribir postales cortas y sencillas, por ejemplo para enviar felicitaciones" / "Soy capaz de escribir notas y mensajes breves y sencillos relativos a mis necesidades inmediatas. Puedo escribir cartas personales muy sencillas, por ejemplo agradeciendo algo a alguien" (Consejo de Europa, 2001). De este modo, se solicitó a los participantes que escribieran un texto breve, aunque de extensión libre, en relación a su persona, presentándose y aportando cualquier tipo de información sobre sí mismos, su entorno o cualquier tema del cual pudieran dar algún tipo de información en aragonés. Asimismo, se les animó a que, en el caso de que se sintieran incapaces de escribir en aragonés, realizaran su redacción en castellano pero introduciendo los rasgos o léxico de la lengua aragonesa que les fuera posible.

Para llevar a cabo el proceso de validación, una vez adaptado el instrumento este se envió a 4 expertos en Didáctica de las Lenguas de la Universidad de Zaragoza y a 4 expertos en enseñanza de la lengua aragonesa en Educación Primaria. Se les solicitó la cumplimentación de un informe en el que se tenían en cuenta: 1) la adecuación de la prueba para evaluar la competencia lingüística en aragonés dentro de un nivel A1 / A2 tomando como referencia el Marco Común Europeo de Referencia para las Lenguas (habilidades de comprensión oral y escrita y expresión escrita) y 2) la adecuación de la prueba a los diferentes contextos sociolingüísticos donde iba a ser aplicada. Una vez recibidos los informes de los evaluadores, se realizaron pequeñas modificaciones. Teniendo en consideración las recomendaciones de los jueces, se realizaron dos cuestionarios: uno en aragonés común o estándar para su aplicación en los centros situados en zonas de empleo histórico pero sin empleo en la actualidad y otro en la variedad propia del territorio en el que se localizaba 
el centro de Tipo 1. Además, las grabaciones para la prueba de comprensión oral también se prepararon por duplicado, respetando del mismo modo la realidad sociolingüística de los centros participantes.

Para la corrección de las pruebas adaptadas del cuestionario original (de tipo cuantitativo) se tomaron como referencia las indicaciones establecidas por del Departamento de Educación, Cultura y Deporte del Gobierno de Aragón (2010) y se obtuvieron las medias para cada grupo en cada uno de los apartados y en el cuestionario completo. Con respecto a la prueba de expresión escrita se consideró suficiente una valoración de tipo cualitativo dado que las diferencias entre el alumnado del centro de Tipo 1 - L1 y de los de Tipo L2 - escolar y Tipo L2 - extraescolar eran extremas. Dado que la evaluación de la competencia escrita resulta compleja al no responder a un modelo de prueba objetiva basada en preguntas cerradas, se consideró oportuno realizar la misma partiendo de un esquema evaluativo en el que se abarcasen todos los aspectos de la escritura. Para poder llevar a cabo la valoración de los escritos se creó una plantilla en la que se plantearon 4 criterios de evaluación con 3 niveles de competencia demostrada (alta, media o baja). Los criterios de evaluación de esta prueba fueron los siguientes: 1) Utilización de vocabulario y sintaxis aragonesa, sin transferencias del castellano; 2) Estructuración de las frases (sintaxis); 3) Conjugación de los verbos; 4) Estructuración / enlace de las partes del texto.

\section{Resultados}

En lo que respecta a la prueba adaptada de la evaluación diagnóstica de la Comunidad de Aragón, se respetó el peso de cada apartado de la matriz que define la competencia lingüística del cuestionario original: competencia en comprensión oral 35/100, competencia en comprensión escrita 35/100 y competencia en gramática 30/100 (Departamento de Educación, Cultura y Deporte del Gobierno de Aragón, 2010).

Teniendo en cuenta esta matriz se calcularon las medias para cada una de las competencias en cada uno de los grupos, dividiendo asimismo el grupo 1 (alumnado de territorio aragonesófono) en dos subgrupos (los que tomaban clases de aragonés y los que no las tomaban). También se obtuvo la media de la prueba completa para todos los grupos. Los resultados quedan recogidos en la Tabla 1.

Tabla 1: Resultados totales y por apartados para cada grupo de participantes.

\begin{tabular}{|l|c|c|c|c|}
\hline \multicolumn{1}{|c|}{ Grupo } & $\begin{array}{c}\text { Comprensión } \\
\text { oral }\end{array}$ & $\begin{array}{c}\text { Comprensión } \\
\text { escrita }\end{array}$ & Gramática & Total \\
\hline Tipo L1 (clases) & 29,53 & 30,91 & 26,11 & 86,55 \\
\hline Tipo L1 (no clases) & 28,63 & 28 & 20 & 76,63 \\
\hline Tipo L2 (escolar) & 32,87 & 32,08 & 11,25 & 76,20 \\
\hline Tipo L2 (extraescolar) & 29,33 & 21,77 & 8,03 & 59,13 \\
\hline
\end{tabular}


En lo relativo a la prueba de expresión escrita, como se ha señalado, la flexibilidad fue máxima, dando gran libertad al alumnado en la temática de la redacción.

Los resultados de esta prueba fueron reveladores. En el caso del grupo de alumnos de Tipo L2 - extraescolar de los 9 estudiantes cuyos resultados se han evaluado 5 de ellos declinaron realizar la prueba alegando no ser capaces de escribir nada en aragonés. Ante la insistencia de la investigadora, dos de ellos intentaron escribir algo, pero su producción se redujo a una sola frase. Así, el Participante E.S.O. escribió "Yo en mi tiempo libre juego", una única frase y en castellano y el Participante A.B.M. escribió: "Me gusta jugar con ma familia", también solo una frase en castellano pero con el posesivo en francés. Entre los que decidieron escribir desde el primer momento, dos de ellos realizaron sus composiciones completamente en castellano, sin ningún rasgo morfosintáctico ni léxico en aragonés. Para finalizar, dos alumnos redactaron textos con algún rasgo en aragonés. Esta es la transcripción de sus escritos:

Participante C.C.B.: "Me clamo C. Yes naxiu en *. Me goyo jue a baloncesto, yes canto y danzo. Yes $a *$ añadas. Mi familia la forma mi mai, mi pai e o mi a hermana. Fin".

Participante: L.P.E.: "Buen diya, me clamo L. tengo * añadas y vivo ta* com mais pais y com mi chirmana y mi chirmano. Yo voy al colegio y después hago los deberes. Cuando termino veo televisión, después ceno, y me voy a dormir”.

En lo que respecta al alumnado del grupo Tipo L2 - escolar los 6 participantes realizaron la prueba, elaborando escritos de extensión considerable. Sin embargo, no puede considerarse que sus producciones estén realizadas con corrección lingüística en aragonés. En cualquier caso, en todas ellas se encuentran rasgos morfosintácticos y léxicos en lengua aragonesa, tal y como podemos comprobar en el texto del siguiente alumno:

Participante A.P.P.: "Buen diya mi nombre ye Alba ye naxiu a *. Tiengo * añadas.

Tiengo una mai qui se encarga de trabaillar de fair as cosas o casa. Sara ye o nombre de mi chirmana ye un poquet revoltosa ye mas chicotona que yo. Tiengo un tío qui se encarga ta faire os cosas o campo. Tiengo una tía qui trabailla a la pista da Astún. Una lola qui ya ye mayor qui se encarga de dar de minchar os animals. Me cuaca faire leer, chugar con mis amigos y con mi chirmana. Se me olvidaba, ta color de mis güellos ye pardo. Mi pelo ye do color castañez y también lo llevo ta siempre largo con coleta".

En contraposición a estos datos, encontramos el caso del alumnado del centro Tipo 1. Tanto el que acude a clases de aragonés como el que no acude realizó la prueba. Las producciones del alumnado que tomaba clases fueron casi perfectas en todos los casos, tal y como se puede comprobar en el texto del siguiente participante:

Participante J.U.M.: "Me clamo J., he * añadas. Nacié lo *2-1*-200*. La mía familia ye la mía mai, lo mío pai y una hirmana. La mía mai se clama N., a 46 añadas y ye de Cataluña. Lo mío pai se clama F. J. y a 44 añadas y ye d'*. La 
mía hermana se clama $J$ y a 11 añadas. Me fa goyo lo fútbol, la pala y chugar. No me cuacan las chodias.

El alumnado de este centro que no acude a clases de aragonés realizó composiciones con bastantes rasgos en esta lengua. Hay que destacar que este grupo, de un total de 4 participantes, además de no tomar clases de aragonés realiza sus actividades cotidianas en un contexto castellanófono. Así, del total de 4 sujetos 2 viven en localidades alejadas al pueblo en el que se encuentra el centro, en las cuales el aragonés ya no se habla (se desplaza a la escuela cada día pero su vida cotidiana se desarrolla en un contexto no aragonesófono), 1 de ellos es hijo de un matrimonio mixto en el que no se ha continuado con la transmisión de la lengua propia y el último de ellos es descendiente de una familia llegada a la localidad hace unos años desde otra provincia, por lo que su contexto es mayoritariamente castellanófono al ser esta la lengua de sus relaciones familiares. Es la composición de este último participante la que vamos a reproducir:

Participante A.B.G.: "Hola me clamo A. he * años. Ne'e naciu en Z. En la mía familia ne hay un mesache que se clama M. otro que se clama $Q$. y una mesacha que se clama B. Me fa gollo jugar al futbol jugar al baloncesto lier cuents $y$ comics chugar al ajedrez y ver la tele".

Estos hechos reflejan una posible influencia positiva del contexto sociolingüístico (aragonesófono) sobre la competencia en aragonés, dado que a pesar de que este alumnado no ha recibido clases de aragonés y cuenta con una situación lingüística familiar completamente castellanófonona, su producción escrita es asimilable a la del alumnado Tipo L2 - escolar.

Para confirmar o desestimar este influjo, se decidió realizar la prueba de competencia escrita a dos alumnos del mismo centro del primer curso del tercer ciclo (dado que todos los de segundo ciclo ya habían participado en la evaluación) que nunca han tomado clases de aragonés pero cuyo contexto familiar es aragonesófono (a pesar de que uno de ellos desciende de un matrimonio mixto). Los resultados fueron similares e incluso mejores que los del alumnado de segundo ciclo que sí toma clases de aragonés, al ser sus textos más ricos desde el punto de vista morfosintáctico y léxico y contener únicamente algunos errores ortográficos. Estas fueron sus redacciones:

Participante J.U.M.: "Me clamo J. y he * añadas. Nacié lo día * de chulio de l'año $2^{* * *}$ en Chaca. So alta, he los güeyos marrons. He lo pelo largo y castaño. La mia mai se clama N. y lo mio pai se clama F.J. pero los suyos amigos lo claman P. So d'*. Lo día de la virxen me visto de * con lo mío trache. M'hirmano que se clama J. tamien se viste con lo trache de lo mio bisagüelo. Me fa goyo fer comella y deporte. De gran quiero estar diseñadora de ropa”.

Participante M.: "So M. y e * añadas e naciu en Chaca, lo dia **-chinero-2***. So alta con lo pelo corto castaño y rizau e unos güellos grans y de color verde y marrons. So divertida, me fa goyo fer comeya, bailar... Mi mai se clama M.J. $a *$ añadas le gusta muito lo teatro. Mi pai a* añadas y ye cocinero. A mi me fa muito goyo vestirme con lo trache *. E dos hirmanos se claman T. a * añadas y M. $a$ * añadas. ASTA LUGO!". 


\section{Discusión}

Los resultados de las pruebas aplicadas parecen confirmar nuestros presupuestos de partida, aunque deben matizarse algunos aspectos. Respecto a nuestro primer presupuesto, 1) La enseñanza actual del aragonés no garantiza una competencia lingüística suficiente entre el alumnado de territorios de empleo histórico de la lengua, podemos decir que los resultados obtenidos podrían ajustarse a este de forma parcial. De este modo, podemos comprobar que todos los grupos participantes, pertenezcan a centros situados en contextos aragonesófonos o no y tomen las clases dentro o fuera del horario escolar, obtienen unos resultados positivos en la compresión del aragonés. De este modo, se podría considerar que todos poseen una alta competencia en la comprensión oral y escrita, si bien en esta última los resultados del alumnado Tipo L2 - extraescolar son inferiores respecto al resto. Esta elevada competencia en las destrezas comprensivas hace que los resultados totales obtenidos por todos los grupos en la prueba de tipo cuantitativo sean de carácter positivo (todos con una puntuación superior a 50/100). Sin embargo, consideramos muy oportuno centrar nuestra atención tanto en el apartado de evaluación de la competencia en gramática, así como de la prueba de expresión escrita que añadimos al cuestionario. Estos datos nos muestran una realidad diferente. En primer lugar, respecto a los resultados de la prueba de gramática del cuestionario, encontramos una clara diferencia entre el alumnado del grupo Tipo 1, tanto si acuden como no a las clases de aragonés, y el resto de los grupos. De este modo, los primeros (Tipo 1 que acuden a clases de aragonés) cuentan con una media muy elevada $(26,11$ / 30) y los segundos (Tipo 1 que no acuden a clases de aragonés) cuentan con un resultado inferior pero también positivo $(20 / 30)$. En el otro extremo se encuentran los participantes de los centros Tipo L2, tanto si toman las clases de aragonés en horario escolar $(11,25 / 30)$ como extraescolar $(8,03 / 30)$, si bien es cierto que los resultados de los primeros son más elevados. En cualquier caso ninguno de los dos grupos alcanza la puntuación media de $15 / 30$.

Estos datos parecen confirmarse a través de la prueba de competencia en la expresión escrita, la cual muestra que el alumnado participante que cursa aragonés en territorios de empleo histórico pero no actual no posee una competencia suficiente para comunicarse en aragonés alcanzando los objetivos similares a un nivel A1/A2 del MCER (Consejo de Europa, 2001). Así, a pesar de que los resultados entre estos participantes sí muestran una diferencia entre aquellos que cursan la asignatura en horario escolar y los que la cursan en horario extraescolar, siendo más positivos los de los primeros, una revisión exhaustiva a sus producciones refleja un intento por reproducir las frases del enunciado del ejercicio (Agora, has de fer tú una descripción de qui yes. Presenta-te, di cuántas añadas tiens, de dó yes naxiu, cómo ye a tuya familia, qué cosas te fan goyo, etz.) sin una correcta flexión de los verbos; así como del vocabulario leído en el cuestionario anterior, sin un buen dominio morfosintáctico y apenas nuevas producciones léxicas aparte de las leídas en la prueba realizada inmediatamente antes.

Gracias a la prueba añadida de expresión escrita hemos podido, asimismo, conocer un hecho que no planteábamos en nuestros presupuestos de partida: parece darse el caso de que la presencia social de la lengua tiene mayor influjo sobre la competencia lingüística del alumnado que la asistencia a las clases de aragonés. Así, en primer lugar, a través de la prueba de gramática del cuestionario se muestra una mayor competencia escrita por parte del alumnado de contexto aragonesófono que no toma clases de aragonés que por parte de 
cualquiera de los dos grupos de contexto no aragonesófono que sí toman clases de esta lengua. Pero, además, la prueba de expresión escrita muestra unas producciones muy similares entre estos dos tipos de alumnado. Además, el peso del contexto sociolingüístico queda refirmado a través de la prueba de expresión escrita realizada a dos alumnos que nunca han tomado clases de aragonés pero cuyo contexto cotidiano es aragonesófono, con sus ricas producciones.

Esto nos lleva a realizar dos consideraciones que, en cualquier caso, no pueden tomarse como definitivas dada la naturaleza exploratoria de nuestro estudio. En primer lugar, podríamos considerar que la asistencia a las clases de aragonés tal y como se desarrollan en la actualidad no es la que garantiza la competencia lingüística en los contextos aragonesófonos, teniendo mayor peso el contexto sociolingüístico. En segundo lugar, los resultados podrían demostrar que la enseñanza del aragonés tal y como se ha venido desarrollando desde 1997 hasta la actualidad no garantiza que el alumnado adquiera una competencia bilingüe, pues presenta grandes carencias en la producción escrita (conocimientos en gramática / morfosintaxis y léxico). Se podría considerar a estos alumnos como bilingües pasivos (Baker, 1997) es decir, personas que poseen una alta competencia para recibir mensajes tanto orales como escritos en la L2 pero no para emitirlos. Sin embargo, tal y como señala Siguán, ni este tipo de sujetos, ni aquellos que poseen "conocimientos de otra lengua que sólo le permiten utilizarla en forma limitada y en determinadas circunstancias" (Siguán, 2001:29), pueden calificarse como bilingües y, por lo tanto, no son garantes de la salvaguarda de la L2.

En lo que respecta a nuestro segundo presupuesto de partida, 2) En los territorios de empleo histórico pero no actual de la lengua, su presencia en el horario escolar influirá sobre el nivel de competencia del alumnado, los resultados obtenidos parecen corroborarlo, aunque consideramos que también deben realizarse algunas matizaciones.

Así, aunque los resultados en comprensión tanto oral $(32,87$ / 35) como escrita $(32,08$ / 35) son más positivos entre este tipo de alumnado si toman las clases de aragonés en horario escolar, cabe señalar que los resultados en estas competencias (29,33 / 35 y 21,77 / 35 respectivamente) en el alumnado que asiste a estas clases en horario extraescolar también pueden considerarse como positivos.

También encontramos diferencias entre estos dos grupos en el apartado de gramática del cuestionario, obteniendo de nuevo el alumnado de Tipo L2 - escolar unos resultados más positivos $(11,25 / 30$ frente a 8,03 / 30). Asimismo, cabe señalar que la totalidad de alumnado Tipo L2 - escolar se enfrentó a la realización de la prueba de expresión escrita sin presentar los inconvenientes, posiblemente relacionados con un nerviosismo derivado de su escasa competencia en aragonés, que sí manifestó el alumnado Tipo L2 - extraescolar. Además, sus producciones presentan más rasgos aragoneses que las del alumnado que toma las clases en horario extraescolar. En cualquier caso consideramos que no debe olvidarse que ninguno de los dos grupos de alumnos del Tipo L2, tomen las clases dentro o fuera del horario escolar, ha demostrado poseer una competencia suficiente en aragonés, siendo ambos superados por el alumnado que no cursa aragonés ni tiene un contexto sociolingüístico cotidiano aragonesófono pero que estudia la Educación Primaria en un centro situado en un territorio de uso habitual de esta lengua.

En resumidas cuentas, los resultados de nuestro estudio piloto parecen dar una primera respuesta a la pregunta que abre este artículo: parece ser que la enseñanza del aragonés tal y como se ha venido desarrollando desde que comenzase a implementarse en 1997 hasta la actualidad no garantiza la salvaguarda de la lengua, siendo el contexto sociolingüístico del alumno el que tiene el peso determinante sobre su competencia en aragonés. 
Todo ello nos lleva a pensar en un futuro incierto para el aragonés, teniendo en cuenta que en las últimas décadas el territorio de utilización de esta lengua ha menguado de forma acelerada, siendo muy escasos los lugares en los que la lengua tiene cierta vitalidad actualmente. En cualquier caso, es oportuno recordar que los resultados de este primer estudio piloto pueden tomarse únicamente como un primer acercamiento y respuesta parcial a la pregunta formulada, por lo que, una vez validado nuestro instrumento y realizada esta primera aproximación, deberemos llevar a cabo de nuevo la experiencia con una muestra más amplia y representativa para poder obtener unas conclusiones definitivas.

\section{REFERENCIAS}

Alcover, C. y Quintana, A. (2000). Plans reguladors d'ensenyament de l'aragonès i el català a l'Aragó. Zaragoza: Rolde de Estudios Aragoneses.

Castán, C. (1997). "Chen que charra l'aragonés benasqués en Benás en 1997”, en Luenga y Fablas, 2, 213-216.

Cenoz, J. (2009). Towards multilingual education. Basque Educational Research from an International Perspective. Bristol: Multilingual Matters.

Comisión de las Comunidades Europeas (2008). Multilingualism: an asset for Europe and a shared commitment. Bruselas. Consultado en: http://ec.europa.eu/languages/documents/2008_0566_ en.pdf, última consulta el 10 de septiembre de 2014.

Consejería de Educación del Gobierno de Aragón (2013). Orden de 14 de febrero de 2013, de la Consejera de Educación, Universidad, Cultura y Deporte, por la que se regula el PrograPrograma integral de bilingüismo en lenguas extranjeras en Aragón (PIBLEA) a partir partir del curso 2013/14. Recuperado de: http://www.educaragon.org/files/Orden_biling\%C3\%BCismo\%2013-14.pdf, última consulta el 10 de septiembre de 2014.

Consejo Escolar de Aragón (2014). Informe 2014 sobre la situación del sistema educativo en Aragón. Recuperado de: http://www.educaragon.org/files/informe_2014_curso_12-13.pdf, última consulta el 10 de septiembre de 2014.

Consejo de Europa. (1992). Carta Europea de las Lenguas Regionales o Minoritarias. Estrasburgo. Consultado en: http://www.lexureditorial.com/boe/0109/17500.htm, última consulta el 10 de septiembre de 2014.

Consejo de Europa. (2008). Council resolution on an European strategy of multilingualism. Bruselas. Consultado en: http://www.consilium.europa.eu/ueDocs/cms_Data/docs/pressData/en/ educ/104230.pdf, última consulta el 10 de septiembre de 2014.

Consejo de Europa. (2010). Guide for the development and implementation of curricula for plurilingual and intercultural education. Obtenido de http://www.coe.int/t/dg4/linguistic/ Source/Source2010_ForumGeneva/GuideEPI2010_EN.pdf, última consulta el 10 de septiembre de 2014.

Consejo de Europa (2001). Marco común europeo de referencia para las lenguas: aprendizaje, enseñanza, evaluación. Recuperado de: http://cvc.cervantes.es/ensenanza/biblioteca_ele/ marco/cvc_mer.pdf, última consulta el 10 de septiembre de 2014.

Cook, V.J. (1992). "Evidence for multicompetence", en Language Learning 42, 557-91.

Cortes de Aragón. (2007). Ley orgánica 5/2007, de 20 de abril. Reforma del Estatuto de Autonomía de Aragón. Recuperado de: http://www.boa.aragon.es/EBOA/pdf/ESTATUTOAUTONOMIA. pdf, última consulta el 10 de septiembre de 2014.

Departameto de Educación, Cultura y Deporte del Gobierno de Aragón (2009). Evaluación de diagnóstico. Educación Primaria. Marco teórico y matriz. Competencia comunicación 
lingüística: inglés. Recuperado de: http://www.evalua.educa.aragon.es/admin/admin_1/ file/DefCBMatrices/IngPRIMarcoTeoricoyMatriz_08-09.pdf, última consulta el 10 de septiembre de 2014.

Departamento de Educación, Universidad, Cultura y Deporte del Gobierno de Aragón. (2014). Orden de 16 de junio de 2014, de la Consejera de Educación, Universidad, Cultura y Deporte, por la que se aprueba el currículo de la Educación Primaria y se autoriza su aplicación en los centros docentes de la Comunidad Autónoma de Aragón. BOCA, 119, 19288-20246, última consulta el 10 de septiembre de 2014.

DGA (Dirección) (2009). 25 años de la Declaración de Mequinenza [Película]. Huesca.

Grosjean, F. (1982) Life with Two Languages: An Introduction to Bilingualism. Cambridge, MA: Harvard University Press.

Hirvonen, V. (2008). " $<$ Out on the fells, I feel like a Sámi $>$ : Is there linguistic and cultural equality in the Sámi”. En Hornberger, N. H. (ed.) Can schools save indigenous languages?, 15-41. London: Palgrave.

Johnstone, R. (2006). "Characteristics of immersion programs”. En García, O. y Baker, C. (eds.) Bilingual Education, 19-32. Clevedon: Multilingual Matters.

Justicia de Aragón. (1993). "Informe anual del Justicia de Aragón, 1993". Zaragoza: BOCA, 25. Lewis, G. (2008). "Current challenges in bilingual education in Wales", en Alia Review, 21, 69-86. Llera, F. (2001). Estudio sociolingüistico de las hablas del Alto Aragón. Euskobarómetro. [Inédito]. López, J. I. (coord). (2013). El aragonés en el siglo XXI. Zaragoza: Fundación Gaspar Torrente. Loredo, X., Fernández, A., Suárez, I. y Casares, H. (2007). "Language use and language attitudes in Galicia". En Lasagabaster, D. y Huget, Á. (eds.) Multilingualism in European bilingual contexts, 40-64, Clevedon: Multilingual Matters.

Mackey, W.F. (1967). Bilingualism as a World Problem. Montreal: Harvest House.

Nagore, F. (2001). "Del bilingüismo diglósico a la sustitución lingüística". En Actas del II Simposio Internacional de Bilingüismo. Universidad de Vigo. Recuperado de: http://webs. uvigo.es/ssl/actas2002/04/11.\%20Francho\%20Nagore\%Lain.pdf, última consulta el 10 de septiembre de 2014.

Ó Laoire, M. (2007). "Language use and language attitudes in Ireland". En Lasagabaster, D. y Huguet, Á. (eds.) Multilingualism in European bilingual contexts. Clevedon: Multilingual Matters, 164-183.

Piquer, I. (2006). "Aprender inglés en la escuela desde los 3 años", en Porta Linguarum, 6, 115-128. Quintana, A. (1985). “QQué aragonés n’a escuela?”, en Rolde, 30, 4-5.

Satué, E. y L'Hotellerie, R. (2011). As crabetas. Libro museo sobre la infancia tradicional del Pirineo. Zaragoza: Prames.

Siguán, M. (2001). Bilingüismo y lenguas en contacto. Madrid: Alianza Editorial.

Skutnabb-Kangas, T., Phillipson, R., Mohanty, A.K. y Panda, M. (2009). Social justice through multilingual education. Bristol: Multilingual Matters.

UNESCO (1996). Declaración Universal de los Derechos Lingüísticos. Obtenido de: http://www. unesco.org/cpp/sp/declaraciones/linguisticos.htm, última consulta el 10 de septiembre de 2014 .

Vila, F. X. (1994). La enseñanza de las lenguas minoritarias en Europa: ensayo de una revisión histórica y sociolingüística. Lletres asturianes: Boletín Oficial de l'Academia de la Llingua Asturiana, 51, 35-46.

Vila, F.X. (2008). "Language-in-education policies in the Catalan language area", en Aila Review, $21,31-48$.

Ytsma, J. (2007). "Language use and language attitudes in Friesland". En Lasagabaster, D. y Huguet, Á. Multilingualism in European bilingual contexts. Clevedon: Multilingual Matters, $144-163$. 